\title{
REALTIME ENVIRONMENTAL MONITORING OF THE GREAT BARRIER REEF
}

\author{
A.B.Ruxton $^{1}$, G.S. Woods ${ }^{l}$, Gilles Gigan ${ }^{2}$, and C.R. Huddlestone-Holmes ${ }^{3}$ \\ School of Engineering, James Cook University, Townsville QLD 4811', School of Mathematics, Physics \\ and IT, James Cook University, Townsville QLD 481 $1^{2}$, Information Technology and Resources, James \\ Cook University, Townsville $Q L D 4811^{3}$
}

\begin{abstract}
The Great Barrier Reef (GBR) is the largest natural feature on earth, stretching more than $2,300 \mathrm{~km}$ along the northeast coast of Australia. It contains a diverse array of species and habitats that are threatened by environmental and human impacts. Monitoring the environmental changes on the GBR would enable scientists, managers and commercial groups to better understand the health of this ecological system. It would also help shape decisionmaking to ensure this important natural asset is protected into the future.

This paper investigates how environmental information can be collected, complied and brought back to the mainland from the GBR. In particular, this paper address the three major components of this type of system; the high speed communication link needed to connect the reef to the mainland, the design of the communication base station (or node) on the reef itself, and the sensor network to be deployed across the reef. It is shown that all three parts of this system involve unique design challenges due to the harsh conditions and the remote location of the region to be monitored. Two key issues, the high speed communication link to the mainland and the power supply for the reef node, are focused on in this paper. A novel method of using the evaporation duct just above the ocean surface to tunnel radio signals beyond the horizon is proposed for a high speed communication link to the mainland. The design of a hybrid wind/ solar supply to power the reef node is also outlined. A monitoring network for use on Davies Reef, approximately $80 \mathrm{~km}$ off the coast of northern Queensland, is used as a case study in this paper.
\end{abstract}

\section{KEYWORDS}

Evaporation duct, ocean communications, radio propagation

\section{INTRODUCTION}

The monitoring of marine processes through collection and analysis of environmental data aids scientific understanding of ecosystems such as the Great Barrier Reef (GBR). Through monitoring it is possible to detect changes in the ecosystem, due to natural phenomenon and occurrences or anthropogenic stresses. Changes caused by human activities must be identified to improve decision-making and management of these natural resources, ensuring sustainable use and continued conservation. Monitoring of the GBR is difficult due to the fact it is a geographically large, complex system with multiple interdependencies between the many environmental parameters of interest. It is therefore useful to observe as many environmental parameters as possible to try and accurately capture these interdependencies.

This type of monitoring will be essential to answer the important questions now facing the GBR. Examples of this could be measuring water currents in a central reef area that splits in two directions. What happens to the nutrients in the water? Another example would be the further understanding of coral bleaching. It is obviously important to monitor water temperature as an indicator of a bleaching event (Berkelmans \& Oliver, 1999; Jones, Berkelmans, \& Oliver, 1997; Lough, 1999), but how do the flow rates and tide heights influence how hot the water gets and how long it remains at an elevated temperature?

Some long term physical environmental monitoring (such as water temperature) from various locations on the GBR has been conducted by the Australian Institute of Marine Science (AIMS) through the use of fixed location weather stations for several years (Berkelmans et al., 2002). In these systems data has been sent back to the mainland via HF radio on a regular basis. However, the amount of data that can be collected has been limited by the low baud rate of existing weather station equipment (Kininmonth et al., 2004) and reliability issues (Contreira et al., 2005). As demand increased for more monitoring, floating buoys have been used to measure other variables where fixed structures are unable to be erected. The main problem with floating buoys is the necessity to store the data locally until such time as it can be physically retrieved. Since this may be infrequent, typically 6 months, this restricts the ability to respond to transient ecological events (Lough, 1999). Furthermore, buoys have finite restrictions on storage and power requirements, limiting the amount of information that can be collected.

The desire of scientists to gain a better picture of the ecosystem dynamics has increased demand for the collection of real time environmental information. The existing methods outlined above are incapable of delivering this, and therefore other methods need to be developed. The alternatives are few, due to the remoteness of the sites of interest 
(up to $100 \mathrm{~km}$ offshore), harsh environmental conditions (salt air, high wind speeds), geographic span of these sites (approx 2,300km long) and the accessibility (boating vessel, helicopter) of the GBR. The key deliverables for future GBR monitoring systems are:

1. to have good reliability (i.e. lose a single collection node and the system still works)

2. the system needs to be easily deployed (i.e. not just on fixed structures), and

3. be able to relay information back to the mainland in real-time.

This paper describes a system developed to pilot a real time sensor monitoring network at Davies Reef approximately $80 \mathrm{~km}$ off Townsville, using high speed microwave communications and wireless sensors in the ocean environment.

\section{MATERIALS AND METHOD}

The materials and method used in design of the prototype system are as follows, and are expanded upon in the sections below. The prototype system consists of an emSolutions microwave radio link operating in the $10.5 \mathrm{GHz}$ region with a data rate of $10 \mathrm{Mbps}$. It has a receive threshold of $92 \mathrm{~dB}$ for a bit error rate (BER) of $1 \times 10^{-6}$, uses a spectral bandwidth of $14 \mathrm{MHz}$ for full duplex communications and QPSK as the modulation scheme. Four 125W Sharp brand ND-L5E6E solar panels, combined with one Southwest Windpower AirX Marine wind turbine, six 100Ah sealed batteries, and one Plasmatronics PL40 solar regulator form the power system. Ambient Systems wireless sensor nodes and commercially available 1-wire sensors measuring humidity and temperature, interfacing with a small form-factor computer motherboard form the sensor network.

\section{ReefGrid}

The ReefGrid concept is a collaboration between AIMS, James Cook University (JCU), and the University of Queensland (UQ) with support funding from the Queensland Parallel Supercomputing Foundation (now Queensland Cyber Infrastructure Foundation) (QCIF) and Data Acquisition Accessibility and Annotation e-Research Technologies (DART) to establish a monitoring system on the GBR. To achieve the goals of ReefGrid, the activities to be covered included sensor networks, high speed communication links, power systems and development of processes for data acquisition, storage and display. A multi-disciplinary approach was therefore required to bring together expertise from different fields of marine science, information technology (IT) and engineering.

The first major step in this collaboration was to set up a pilot monitoring system on Davies Reef, allowing a central collection point for environmental data to be transmitted back to the mainland. This system would support the running of the sensor network and microwave link through a hybrid solar/wind power system. Once this link was proven, the vision was to extend this concept to a network covering other areas of the reef. There were three main components to this system - the base node (tower, electronics and power supply), the sensor network collecting the environmental information and the microwave link back to shore.

\section{Base Node}

Due to existing infrastructure, suggested locations for the base nodes in the ReefGrid system are AIMS weather station towers. For this particular study, work centred on developing a base node on Davies Reef. This tower has sufficient room to support the equipment needed for the system, and is in close proximity to AIMS and JCU. Modifications to the tower were completed by AIMS engineering staff to support solar panels, a wind turbine, microwave antenna dish, battery bank and communications electronics.

The base node physically houses the microwave link and the controlling computer for the sensor network. As the equipment would be installed on a tower exposed to the elements, consideration of the type of housing was of prime importance. Sealing the electronics to protect them from the elements was therefore essential and the enclosure had to be flexible enough to allow additions and modifications in future. A modified sealed stainless steel box with a perspex panel inserted in its base provides easier access when installing flanges and cables in future upgrades. All cables and connectors were rated at IP66 or better to ensure maximum retardation of foreign matter into the junctions. The antenna mounts for the microwave dish and frame for the solar panels were galvanised to minimise corrosion. 


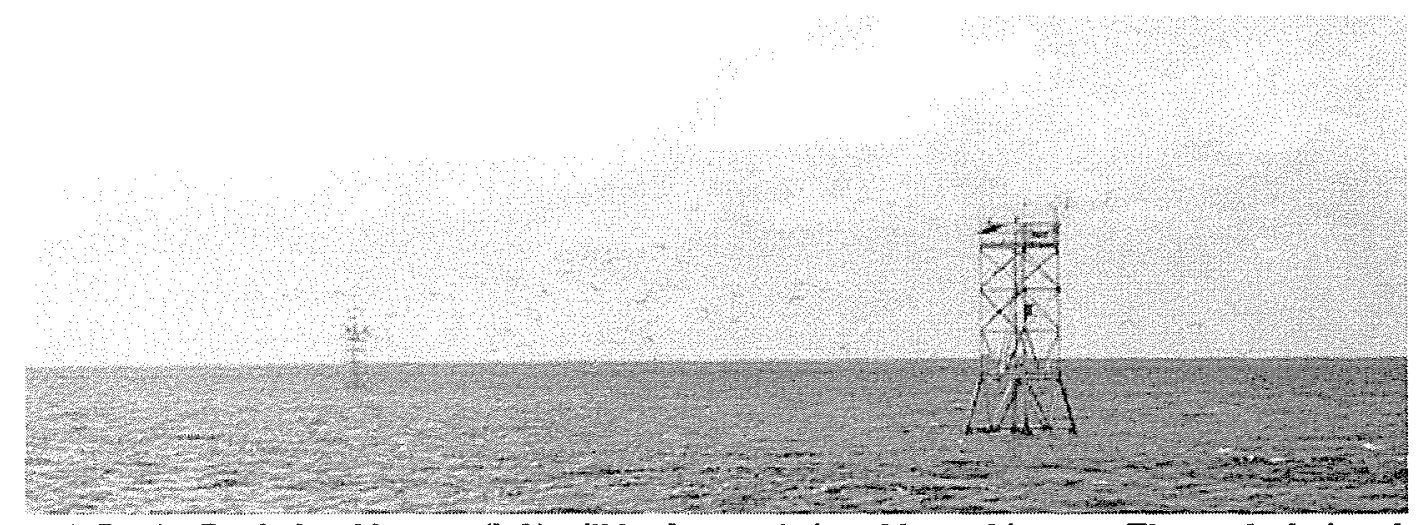

Figure 14: Davies Reef: the old tower (left) will be decommissioned later this year. The newly designed tower (right)

This base node required a self contained power system to maintain operation. Due to the location on the GBR, wind and solar sources were picked as the best environmentally sustainable methods to deliver power to run the base node. Both have their own advantages and disadvantages in this case. A wind turbine should be effective on the water due to a lack of any significant obstructions, but suffers from being a mechanical structure working in an inhospitable environment. Solar cells overcome the mechanical limitations of the wind turbine, but are susceptible to fouling from sea birds which use structures like the tower to roost. Wind speed profiles were obtained from AIMS for Davies Reef and used in a software application called HOMER (NREL) to determine the solar capacity and battery sizes required. Results from this suggest wind speeds would be sufficient to power the system the majority of days throughout the year. The final design however allowed the system to be powered by solar cells alone, to cover those few occasions where low wind speed would not be sufficient. In the event of power issues, the base controller will systematically switch off devices for a set period of time, until sufficient power is available to restart. Data storage facilities on-board ensure information collected would not be lost during an event of this nature.

\section{Microwave Link}

To meet the objectives of ReefGrid, information needed to be transferred at high speeds. The small volumes of data acquired from temperature sensors and the like would not cause delays in system response. However, if real time video footage were to be collected as planned, a much higher speed link would be needed. Therefore the key factor to achieve real-time performance required a novel method of transmission from the GBR to the mainland.

High data capacity radio systems are commercially available and are used in a variety of applications, from linking MANs (Metro Area Networks) to providing building-to-building high speed connections. However, using these systems for distances of anything over a few kilometres normally requires a structure or tower at each end with an elevation high enough to overcome the curvature of the earth. For the distances being considered in this application $(80 \mathrm{~km})$, using conventional ray tracing techniques shows that a structure height of more than $100 \mathrm{~m}$ would be required. This is obviously not practical for environmental, aesthetic and maintenance reasons. Satellite transmission of information is possible, but is an expensive alternative due to the need to transmit information continuously and at fast data rates.

One method identified for long-range signal propagation is by a phenomenon called ducting. This phenomenon is a naturally occurring condition above the surface of the ocean where the rapidly changing humidity from the ocean's surface to the ambient air temperature forms a humidity or evaporation "duct". The height of this duct can vary from being higher than 30 metres (Hitney, Richter, Pappert, Anderson, \& Baumgartner, 1985), to as small as a few metres. There is strong correlation between duct height and the range of propagation of a radio signal (Babin, Young, \& Carton, 1997; Brooks, Goroch, \& Rogers, 1999; Burk, Haack, Rogers, \& Wagner, 2003; Hitney, Richter, Pappert, Anderson, \& Baumgartner, 1985). Many previous studies have been done to characterise this duct through modelling (Sirkova \& Mikhalev, 2006). Work has been conducted at James Cook Unversity to empirically determine the effects of this phenomenon in tropical regions and compare it to common models (Kerans, Kulessa, Lensson, French, \& Woods, 2002, 20 - 22 February; A. Kulessa, Heron, \& Woods, 1998, 27-29 April; A. S. Kulessa, Woods, Piper, \& Heron, 1998). Modelling the anticipated response of signals at certain frequencies in the duct has shown the potential of using this medium for long distance communications, requiring little elevation above sea level for the transmitting and receiving dishes (Palazzi, Woods, Atkinson, \& Kininmonth, 2005, 21-24 November). There has also been work to determine the expected availability of the duct for a specified link range (Woods, Palazzi, Kulessa, \& Maskell, 2006, 16-19 May).

This work showed that high-speed communications over the ocean is possible for the distances required and at the elevation height available at Davies Reef. Based on these results, an off-the-shelf communications link operating at 
the required frequency range of $10.5 \mathrm{GHz}$ was purchased (emSolutions). The link has a data capacity of 20Mbps, and a receive threshold of $82 \mathrm{~dB}$ for a bit error rate (BER) of $1 \times 10^{-6}$. However, although experimental and simulated results have examined signal amplitude, no tests (to the authors' knowledge) have been performed using real data modulated on these signals. It was therefore decided to apply a convolutional error correction algorithm to the system, increasing the sensitivity by up to a further $10 \mathrm{~dB}$. The trade off was a reduction in throughput rate; therefore an effective $10 \mathrm{Mbps}$ link was deployed. This bandwidth was still sufficient to transport all the information from the base node back to the mainland in real time, at least in the prototype system being developed here.

The equipment was designed for traditional wireless communications deployment, and as such the hardware casings were standard 19U rack width for internal installations. This also meant the ventilation relied on convective cooling only, as standard indoor installations are in air-conditioned environments. This problem had to be addressed, as the maximum operating temperature of the unit was expected to be as much as 50 degrees Celsius when exposed to the sun. These issues were taken into account for the Davies Reef end. The layout of equipment in the base node housing was arranged so components that dissipated the most heat were spaced well apart. Two $12 \mathrm{~V}, 8.5 \mathrm{~cm} 130 \mathrm{~mA}$ DC cooling fans were also installed on a dividing wall to allow circulation of air in the sealed housing, removing pockets of higher temperatures. A sensor was also installed in the housing to track this temperature. Shade to the base node from the sun on the tower platform was also necessary, to help lower the overall ambient heat.

Although not an ideal fit for the project, the equipment was selected due to its ready availability, operation within the frequency of interest, and the high-speed transmission rate of the link. The experimental results obtained from this equipment are expected to help correlate predicted model results, and contribute to determining the characteristics that affect the ability to use the duct as a communications medium.

\section{Sensor Network}

In order to collect information within the marine environment, small and unobtrusive instruments must be used so as not to interfere with or disturb the natural habitat. To achieve environment monitoring on the GBR a range of technical and ecological issues need to be considered. Due to the majority of the desired environmental information being under the ocean surface, the instruments need to be small and portable, unobtrusive to the ecosystem, its inhabitants and other users, and have the ability to communicate over several hundred meters of water. Small wireless sensors that collect and transmit information back to a central system was a suitable choice. Sensors using an air medium to transmit information were chosen in this application, but in future it may be possible to send some information using underwater transmission. The main limitations of this technology are the low data rates possible (300 baud) and the interference to and from other marine users both living (whales, dolphins, shrimp) and man made (boats, submarines). AIMS researchers have collaborated with Ambient Systems, a Netherlands-based company who specialise in low power, wireless communication sensors to meet the stated requirements. The advantages of the Ambient Systems sensor nodes include small size (thus low environmental impact) and modularity in creating ad-hoc networks of sensors, which made them the best fit to the pilot system. However, the exercise of having potentially hundreds of these within an area of interest could be cost prohibitive. An approach of using less expensive sensors in conjunction with these was therefore employed.

Ambient System nodes (Ambient_Systems) allowed connection of between 4 and 6 different sensors that transmitted information wirelessly through the ad-hoc network periodically, sending readings every 15 seconds. The nodes are fully programmable. To supplement the use of the Ambient System sensors, commercially available 1 wire sensors were also employed as a low cost, low power alternative. A single, 1 wire sensor costs less than AUD $\$ 5$, compared with between $€ 15-40$ Euro for an Ambient System wireless sensor. This supplement of sensors allows for a more economical deployment across the area of interest.

The main computer board that collates the information from the sensor network uses a Linux operating system, receiving information from the sensors through both USB and serial connections. Data is stored on board in a buffer using an SD memory card at the same time as being sent to the microwave communication link using TCP/IP protocol over an Ethernet connection and a small switch.

Although not implemented to its fullest extent at this time, the technology chosen for this application will initially be based on wireless sensor technology. Ad-hoc sensor networks have recently matured as a means of measuring environmental parameters over a space where the nodes may move or sometimes fail and are therefore well suited to this application. The main problem with using the technology in this case was the harsh conditions that would be experienced by the nodes. Nodes need to be fully waterproof and immune to problems of fouling and tangling with natural and man-made structures. Some problems of current concern include interference with fishermen and boats, and the aesthetic issues of locating possibly hundreds of nodes across a reef system. 


\section{Hybrid Solar / Wind Power System}

The design of the power supply for the Davies Reef Sensor Network provided several challenges. The station is remote, within a harsh marine environment, and needed to produce about $3.12 \mathrm{kWh}$ a day in the worst case scenario based on the manufacturers ${ }^{1}$ equipment ratings. These values assume the microwave link running 24 hours a day. Of all the system components, the microwave link used the most power at $65 \mathrm{~W}$. Designing the system to turn the microwave link on and off (depending on demand) would further reduce the requirements of the overall system.

The design of the power system was done with the assistance of HOMER (NREL), a modelling package that allowed the evaluation of design options based on user input, principally from a financial point of view. In this case however, the authors were practically constrained by factors other than financial considerations; therefore HOMER was used mostly to test different system designs. HOMER allowed the user to input energy resource information such as wind and solar data. AIMS had wind data recorded every half hour for Davies Reef from 1998 to 2003. HOMER could take wind data either as monthly or hourly averages. The AIMS half hourly data was re-sampled to hourly data and input into HOMER. It was found that the annual average wind velocity varied between $6.91 \mathrm{~ms}^{-1}$ (2001) and $7.63 \mathrm{~ms}^{-1}$ (1999). Daily Solar Radiation data as monthly averages were also obtained from NASA's Surface meteorology and Surface Energy website (SmaSE). HOMER calculated hourly data based on these monthly averages. The NASA website also had daily averages for each year from 1983 to 1993 . The annual daily average for this period ranged between $5.90 \mathrm{kWh} / \mathrm{m}^{2} /$ day and $6.23 \mathrm{kWh} / \mathrm{m}^{2} /$ day.

To test various system designs, a worst case load scenario of 130 Watts continuous power consumption was assumed with a $50 \%$ duty cycle for the microwave link. Wind data for the year 2003 was used (average of $7.12 \mathrm{~ms}^{-1}$ ) with sensitivity analysis over a range of $6.5 \mathrm{~ms}^{-1}$ and $7.5 \mathrm{~ms}^{-1}$ (HOMER scales the data for these sensitivity analyses, maintaining the overall distribution of the data). The monthly average solar data was used with a sensitivity analysis range of $5.90 \mathrm{kWh} / \mathrm{m}^{2} /$ day to $6.07 \mathrm{kWh} / \mathrm{m}^{2} /$ day. Components considered for the system were one or two Southwest Windpower AirX Marine wind turbines, up to four 125 Watt solar panels, and up to 600Ah of battery capacity. Sensitivity of the system to a loss of efficiency in the solar panels as a result of fouling (by either bird droppings or salt build up) was also checked, with efficiencies of $60 \%, 75 \%$ and $90 \%$ considered. Scenarios with $0 \%, 1 \%, 2 \%$ and $5 \%$ capacity shortage were also analysed.

In the worst case scenario, a maximum load combined with minimum wind and solar power (radiation and efficiency) found the optimum system had four solar panels, two AirX turbines, and 600 Ah of battery capacity. This system still had $2 \%$ ( 7 days) of unmet demand. When the system was run with the microwave at $50 \%$ duty cycle, $100 \%$ availability could be achieved with three solar panels, two AirX wind turbines and 600Ah of battery capacity. Increasing the efficiency of the solar panels to $75 \%$ made a four solar panel, single AirX wind turbine and $400 \mathrm{Ah}$ battery system. This was the most favourable system, however there was still $2 \%$ unmet demand at maximum load and $100 \%$ availability with the microwave at $50 \%$ of its duty cycle. The sensitivity analysis showed that the optimum system had either three solar panels and two wind turbines or four solar panels and one wind turbine, depending on solar panel efficiency, total solar radiation, and average wind speed.

A solar power regulator was required to regulate the output of the solar panels to provide optimum charging characteristics for the batteries. A Plasmatronics PL40 (Plasmatronics) solar regulator was chosen. The charge cycle of this device could be fully programmed, and allowed the output of the solar panels and wind turbine to be monitored. It could also observe the condition of the battery bank. The AirX Marine wind turbine had its own inbuilt regulator, so this would only be used to top up the solar panel output.

The circuit design for the power supply was relatively simple. The battery bank was $12 \mathrm{~V}$ so the solar panels and wind turbine were attached in parallel. Six, 100 Ah batteries were also set up in parallel, each battery with its own circuit breaker. Diodes were used to protect the wind turbine from a reverse current. A shunt and proprietary monitoring cable from Plasmatronics were used to monitor the wind turbine output.

Ease of installation and maintenance issues were a prime consideration, therefore the system installed had four 125 Watt solar panels, a single AirX Marine wind turbine and six 100Ah batteries. This system provided the best compromise for available power, cost, ease of installation and future maintenance.

\section{CONCLUSION}

A prototype system to allow environmental monitoring on the GBR was described. The major components that make up a single communication node including the power supply, sensor network and high speed microwave link

\footnotetext{
${ }^{1}$ The equipment in the base node was tested using a Powertech MP3090 switching mode DC regulated power supply. The power draw appeared to be closer to $80 \mathrm{Watts}$ or $2 \mathrm{kWh} /$ day.
} 
were discussed. The many challenging aspects of the environment where this equipment was to be deployed meant a purpose built device needed to be designed. Unique designs were developed for each of the stages that make up this system.

The prototype system consists of an emSolutions $10.5 \mathrm{GHz}$ radio link with a data rate of $10 \mathrm{Mbps}$, four $125 \mathrm{~W}$ Sharp brand solar panels, one Southwest Windpower AirX Marine wind turbine, six 100Ah sealed batteries, and one Plasmatronics PL40 solar regulator. It also consists of Ambient Systems wireless sensor nodes and commercially available 1-wire sensors interfacing with a small form-factor computer motherboard.

The initial focus of the ReefGrid system is to provide monitoring for scientific purposes. This system will allow collection of wind speed, water temperature at multiple depths and humidity to form a profile of reef environment dynamics. It is anticipated water column temperature readings will give scientists greater resolution in determining how temperature variations affect the ecosystem resulting in phenomenon like coral bleaching. Video images will also allow observations for periods much longer than currently possible with diving or underwater vehicles and give scientists observations in real time, information that until now has never been available.

It will be possible to collect different types of information through the sensor network, depending on what variables are of interest. It is anticipated the acquisition of real-time data will help develop better models for prediction of ecological events such as coral bleaching, as well as providing cross-validation for existing methods of sea surface temperature (SST) data collection via satellite. Using the evaporation duct as a transmission medium has implications for other applications, such as radar detection and off-shore visual monitoring of shipping routes.

For now, this new level of environmental monitoring will permit an intimate study of the GBR and will help scientists form a better picture of the ecological processes at work in this environment. With better information and understanding, these groups will provide better data to decision makers relating to marine management policy.

\section{REFERENCES}

1. Ambient_Systems. Ambient Systems. Retrieved August 24, 2006, from http://www.ambientsystems. net/ambient/index.htm

2. Babin, S. M., Young, G. S., \& Carton, J. A. (1997). A new model of the oceanic evaporation duct. Journal of Applied Meteorology, 36(3), 193-204.

3. Berkelmans, R., Hendee, J. C., Marshall, P. A., Ridd, P. V., Orpin, A. R., \& Irvine, D. (2002). Automatic weather stations: Tools for managing and monitoring potential impacts to coral reefs. Marine Technology Society Journal, 36(1), 29-38.

4. Berkelmans, R., \& Oliver, J. K. (1999). Large-scale bleaching of corals on the Great Barrier Reef. Coral Reefs, 18(1), 55-60.

5. Brooks, I. M., Goroch, A. K., \& Rogers, D. P. (1999). Observations of strong surface radar ducts over the Persian Gulf. Journal of Applied Meteorology, 38(9), 1293-1310.

6. Burk, S. D., Haack, T., Rogers, L. T., \& Wagner, L. J. (2003). Island wake dynamics and wake influence on the evaporation duct and radar propagation. Journal of Applied Meteorology, 42(3), 349-367.

7. Contreira, D. B., Rodrigues, F. S., Makita, K., Brum, C. G. M., Gonzalez, W., Trivedi, N. B., et al. (2005). An experiment to study solar flare effects on radio-communication signals. In Space Weather (Vol. 36, pp. 24552459). Oxford: Elsevier Science Ltd.

8. DART. Dataset Acquistion Accessibility and Annotation e-Research Technologies. Retrieved 12 September, 2006, from htp://www.dart.edu.au/

9. emSolutions. em Solutions. Retrieved 5 September, 2006, from http:/www.emsolutions.com.au

10. Hitney, H. V., Richter, J. H., Pappert, R. A., Anderson, K. D., \& Baumgartner, G. B. (1985). Tropospheric Radio Propagation Assessment. Proceedings of the leee, 73(2), 265-283.

11. Jones, R. J., Berkelmans, R., \& Oliver, J. K. (1997). Recurrent bleaching of corals at Magnetic Island (Australia) relative to air and seawater temperature. Marine Ecology-Progress Series, 158, 289-292.

12. Kerans, A. J., Kulessa, A. S., Lensson, E., French, G., \& Woods, G. S. (2002, 20 - 22 February). Implications of the evaporation duct for microwave path design over tropical oceans in Northern Australia. Paper presented at the Workshop on the Applications of Radio Science (WARS02), Leura, Australia.

13. Kininmonth, S., Bainbridge, S., Atkinson, I., Gill, E., Barral, L., \& Vidaud, R. (2004). Sensor Networking the Great Barrier Reef. Spatial Sciences Institute Journal (QLD)(October 2004), 35-39.

14. Kulessa, A., Heron, M. L., \& Woods, G. S. (1998, 27-29 April). Refractivity variations in the tropical Australian marine environment. Paper presented at the URSI Commission F CLIMPARA '98 - Open Symposium on Climatic Parameters in Radiowave Propagation, Ottowa, Ontario, Canada.

15. Kulessa, A. S., Woods, G. S., Piper, B., \& Heron, M. L. (1998). Line-of-sight EM propagation experiment at $10.25 \mathrm{GHz}$ in the tropical ocean evaporation duct. Iee Proceedings-Microwaves Antennas and Propagation, 145(1), 65-69.

16. Lough, J. M. (1999). Sea surface temperatures on the Great Barrier Reef : a contribution to the study of coral bleaching: Great Barrier Reef Marine Park Authority. 
17. NREL. HOMER - The Micropower Optimization Model. 2.19. Retrieved 19 July, 2006, from http//www.nrel.gov/homer/

18. Palazzi, C. M., Woods, G. S., Atkinson, I., \& Kininmonth, S. (2005, 21-24 November). High Speed Over Ocean Radio Link to Great Barrier Reef. Paper presented at the IEEE TENCON'05, Melbourne, Australia.

19. Plasmatronics. Plasmatronics. Retrieved 10 September, 2006, from http $/ /$ www.plasmatronics.com.au/

20. QCIF. Queensland Cyber Infrastructure Foundation. Retrieved 11 September, 2006, from http//www gcifedu.au/industry/SensorNet.htm

21. Sirkova, I., \& Mikhalev, M. (2006). Parabolic wave equation method applied to the tropospheric ducting propagation problem: A survey. Electromagnetics, 26(2), 155-173.

22. SmaSE. Surface meteorology and Solar Energy. Retrieved 19 July, 2006, from http:/leosweb.larc.nasa.gov/sse/

23. Woods, G. S., Palazzi, C. M., Kulessa, A., \& Maskell, D. L. (2006, 16-19 May). ReefGrid-A Communication Network on the Great Barrier Reef. Paper presented at the IEEE Oceans '06 Asia Pacific, Singapore. 